\title{
Assessing Directed Information as a Method for Inferring Functional Connectivity in Neural Ensembles
}

\author{
Kelvin So*, Michael Gastpar, Member, IEEE, Jose M. Carmena, Senior Member, IEEE
}

\begin{abstract}
Neurons in the brain form complicated networks through synaptic connections. Traditionally, functional connectivity between neurons has been analyzed using simple metrics such as correlation, which do not provide direction of influence. Recently, an information theoretic measure known as directed information has been proposed as a way to capture directionality in the relationship, thereby moving towards a model of effective connectivity. This measure is grounded upon the concept of Granger causality and can be estimated by modeling neural spike trains as point process generalized linear models. However, the added benefit of using directed information to infer connectivity over conventional methods such as correlation is still unclear. Here, we propose a novel estimation procedure for the directed information. Using physiologically realistic simulations, we demonstrate that directed information can outperform correlation in determining connections between neural spike trains while also providing directionality of the relationship, which cannot be assessed using correlation.
\end{abstract}

\section{INTRODUCTION}

$\mathrm{N}$ EURAL ensembles form complex, interconnected networks that interact through synaptic connections. The study of brain connectivity has traditionally been divided into anatomical connectivity and functional connectivity (along with the related notion of effective connectivity). Anatomical connectivity refers to the structural layout of synaptic connections, measured using staining and fiber tracing techniques [1]. Functional connectivity refers to the temporal correlations between neurophysiological events, such as action potentials or hemodynamic response, regardless of the anatomical connections. A closely related concept, effective connectivity, specifically characterizes the influence that one neural system exerts on another based on an a priori model of the connectivity [1]. For in situ recordings, typically only functional and effective connectivity can be measured due to the difficulty of measuring anatomical connections in vivo.

In the past few decades, a number of techniques have been developed for inferring functional connectivity for

Manuscript received April 15, 2011. This work was supported by the National Science Foundation (NSF) under award CMMI-0941343 to MG and JMC.

K. So* and M. Gastpar are with the Department of Electrical Engineering and Computer Sciences, University of California, Berkeley, CA $94720 \quad$ USA (e-mail: sokelvin@eecs.berkeley.edu; gastpar@eecs.berkeley.edu).

J. M. Carmena is with the Department of Electrical Engineering and Computer Sciences, the Program in Cognitive Science and the Helen Wills Neuroscience Institute, University of California, Berkeley, CA 94720 USA (email: carmena@eecs.berkeley.edu) intracortical recordings, electroencephalography (EEG), positron emission tomography (PET) and functional magnetic resonance imaging (fMRI). These include crosscorrelation [2], spectral coherence [3] and mutual information [4]. On the other hand, models for effective connectivity, such as structural equation modeling [5], dynamic causal modeling [6] and Granger causality based approaches [7], have been extensively applied to EEG, PET and fMRI studies only. For spike train data, the directed transfer function (DTF) method was first proposed by Kaminski et al. [8] as a way to model effective connectivity. More recently, this problem was addressed by Quinn et al. [9] using an information-theoretic measure known as directed information. This is a promising approach that is consistent with the concept of Granger causality and can be used to assess Granger causality graphs of stochastic processes [10]. However, the efficacy of the directed information method compared to conventional methods (e.g. correlation) for inferring connectivity in spike train data has yet to be determined. Here, we present a novel approach to estimating the directed information and demonstrate that this procedure results in higher performance than correlations in detecting connections in simulated spike trains. We also illustrate scenarios where the directionality of the connection given by directed information can provide additional information about the neuronal network that is not captured using correlations.

\section{Methods}

\section{A. Directed Information}

The directed information is a modified version of the wellknown mutual information that better captures the capacity of a communication channel in the presence of feedback [11]. Conceptually, the directed information is based on the same principle as Granger causality by measuring how much better we can predict a process $\mathrm{Y}$ if past information of a process $\mathrm{X}$ is given. This metric is particularly attractive for analyzing connectivity in neural ensemble data for two reasons. Firstly, directed information is designed to characterize channels with feedback and since a neuron's spiking activity is strongly influenced by its past spiking activity (e.g. refractory period), a neuron can be interpreted as a feedback system transmitting to another neuron. Hence, directed information is well suited for capturing connectivity between neurons. Secondly, directed information measures the effect of having past information of another process. This means that if the connection between two neurons $\mathrm{X}$ 


\section{Two-neuron}

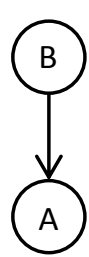

Three-neuron

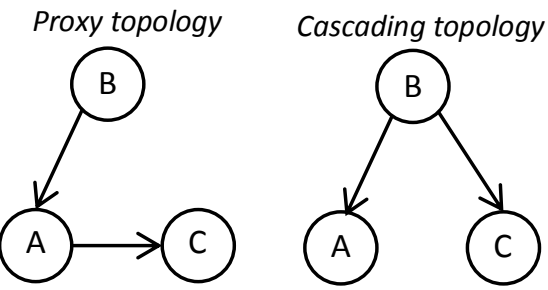

Fig. 1. Two-neuron and three-neuron topologies simulated. Arrows indicate preset influences, i.e. an arrow from neuron B to A means that neuron B's spiking activity is used as input to generating neuron A's spiking activity.

and $\mathrm{Y}$ is unidirectional, as in the activity of neuron $\mathrm{Y}$ is affected by the past activity of neuron $\mathrm{X}$, but neuron $\mathrm{X}$ is not affected by neuron $\mathrm{Y}$, then directed information will only capture the effect of $\mathrm{X}$ on to $\mathrm{Y}$, but not the reverse.

Formally, the directed information between two stochastic processes $\mathrm{X}$ and $\mathrm{Y}$ is defined as:

$$
I\left(X^{N} \rightarrow Y^{N}\right) \triangleq E\left[\sum_{i=1}^{N} \log \frac{P_{Y_{i} \mid Y_{1}^{i-1}, X_{1}^{i}}\left(Y_{i} \mid Y_{1}^{i-1}, X_{1}^{i}\right)}{\left.P_{Y_{i} \mid Y_{1}^{i-1}\left(Y_{i} \mid Y_{1}^{i-1}\right)}\right]}\right.
$$

where $Y_{1}^{n}$ represents the $n$-tuple $\left[Y_{1}, \ldots, Y_{n}\right]$, and $X_{1}^{n}$ represents $\left[X_{1}, \ldots, X_{n}\right]$. Since this quantity scales with $\mathrm{N}$, we define a normalized quantity:

$$
\mathcal{J}(X \rightarrow Y) \triangleq \lim _{N \rightarrow \infty} \frac{1}{N} I\left(X^{N} \rightarrow Y^{N}\right)
$$

Assuming that the processes $\mathrm{X}$ and $\mathrm{Y}$ are finite-memory Markov chains that are stationary and ergodic, it can be shown that a sample average of the log-likelihood ratio will converge almost surely to the normalized directed information. All subsequent computations will use the normalized directed information.

\section{B. Model fitting}

Due to the high dimensionality of the conditional distributions in Eq. (1), we model the spiking activity of a neuron Y using a generalized linear model (GLM) for point processes [12]. Under this model, a neural spike train can be viewed as a Poisson process with a time-varying firing rate that depends on the past spiking activity of itself (intrinsic history) and another neuron (extrinsic history). The conditional intensity and likelihood functions can be written as:

$$
\begin{gathered}
\log \lambda_{i}=\alpha_{0}+\sum_{j=1}^{J} \alpha_{j} Y_{i-j}+\sum_{k=1}^{K} \beta_{k} X_{i-(k-1)} \\
P_{Y_{i} \mid Y_{1}^{i-1}, X_{1}^{i}}\left(Y_{i} \mid Y_{1}^{i-1}, X_{1}^{i}\right)=\frac{\lambda_{i}^{Y_{i}}}{Y_{i} !} e^{-\lambda_{i}}
\end{gathered}
$$

where $\mathrm{X}$ and $\mathrm{Y}$ are as defined above, $\alpha_{0}, \ldots, \alpha_{\mathrm{J}}, \beta_{1}, \ldots, \beta_{K}$ are estimated parameters, $\mathrm{J}$ and $\mathrm{K}$ are the lengths of the intrinsic and extrinsic histories respectively. For a given $\mathrm{J}$ and $\mathrm{K}$, the parameters can be readily estimated using maximum likelihood. However, in practice, $\mathrm{J}$ and $\mathrm{K}$ are not known a priori and must be estimated. To address this, Quinn et al. [9] use a model selection technique, minimum description length (MDL), to estimate $\mathrm{J}$ and $\mathrm{K}$. If the estimated $\mathrm{K}$ is zero, then no connection is detected since $\mathrm{K}$ represents the length of the extrinsic history. For actual implementation,

we used a $5 \mathrm{~ms}$ binning in accordance with [9], and searched over pairs of $\mathrm{J}, \mathrm{K}$ chosen from $\{0,5,10,20\}$ to cover spiking histories up to $100 \mathrm{~ms}$. The same procedure is used to estimate the likelihood without conditioning on $\mathrm{X}$, except $\mathrm{K}$ is enforced to be zero.

\section{Model fitting with smoothness constraint}

Under the above approach, the number of parameters that need to be estimated $(\mathrm{J}+\mathrm{K}+1)$ can become unwieldy. Hence, it may be desirable to impose smoothness constraints over the set of $\alpha$ 's and $\beta$ 's to reduce the number of parameters. Here, we propose the constraint that the set of $\alpha$ 's and $\beta$ 's lie in a subspace spanned by a set of raised cosine functions [13],[14]. With this constraint, the modified conditional intensity function is given by:

$$
\begin{aligned}
\log \lambda_{i}= & \hat{\alpha}_{0}+\sum_{l=1}^{L} \hat{\alpha}_{l}\left\langle b_{l}^{i n t} \cdot Y_{i-R}^{i-1}\right\rangle \\
& +\sum_{m=1}^{M} \hat{\beta}_{m}\left\langle b_{m}^{e x t} \cdot X_{i-R}^{i-1}\right\rangle
\end{aligned}
$$

where $b_{l}^{\text {int }}$ and $b_{m}^{\text {ext }}$ are the sets of raised cosine basis functions of length $\mathrm{R}$ modeling the intrinsic and extrinsic history effects respectively. $\langle\cdot\rangle$ denotes dot product. With this constraint, the number of parameters that need to be estimated is simply $\mathrm{L}+\mathrm{M}+1$, where $\mathrm{L}$ and $\mathrm{M}$ can be chosen to be in general smaller than $\mathrm{J}+\mathrm{K}+1$. In our implementation, we chose $\mathrm{L}=10, \mathrm{M}=4$ with basis functions that cover spiking histories up to $100 \mathrm{~ms}$. Similar to above, we remove the terms involving $X$ to estimate the likelihood without conditioning on $\mathrm{X}$. To determine the significance of the estimated directed information, we employ two validation methods. First, we use cross-validation to assess the fitting. The parameters are fitted to a training set, which is subsequently used to calculate the likelihood of a separate testing set. If the model that conditions on $\mathrm{X}$ results in a lower likelihood than the model without $X$, then conditioning on $\mathrm{X}$ does not improve our ability to predict $\mathrm{Y}$, and the directed information is set to zero. Secondly, we estimate $95 \%$ confidence intervals on the computed directed information [9]. If the interval contains zero, the directed information is set to zero.

\section{Simulation set up}

We simulated neural spiking activity using a sparse generalized Laguerre-Volterra model (SGLVM), which is a nonlinear, multi-input, single-output model used to model activity in the hippocampus [15]. We chose this nonlinear model to avoid biases from similarities in the directed information model estimation and simulation method. The spike trains are simulated using 1st-order and 2nd-order kernel parameters fitted from experimental data in order to produce physiologically realistic simulations. These kernel parameters are used to relate the spiking activity of one neuron to the probability of spiking in another.

To thoroughly compare the effectiveness of directed information versus correlation, we chose six different sets of 1st-order and 2nd-order kernel parameters, for two-neuron and three-neuron topologies (Fig. 1). For each parameter set, 
A Sample spike trains

A

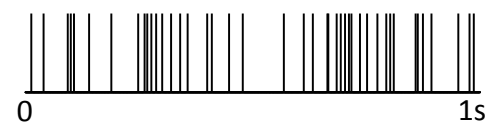

B

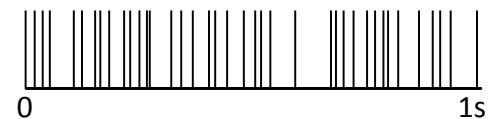

B

$B-A$

Binning/Correlation

$B \rightarrow A$

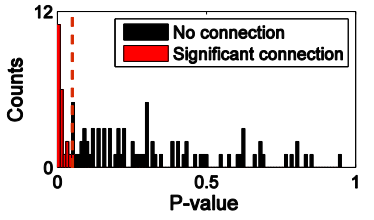

C

CrossVal-DI
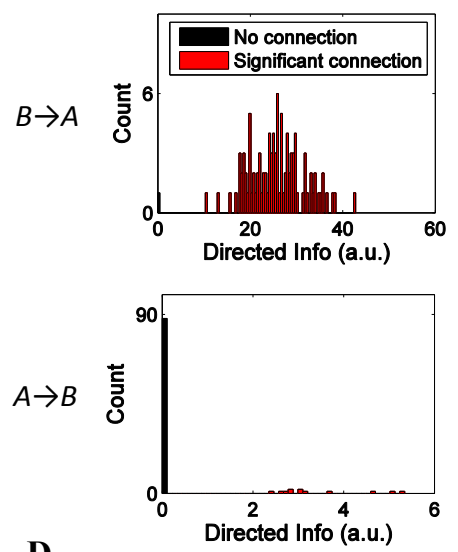

D

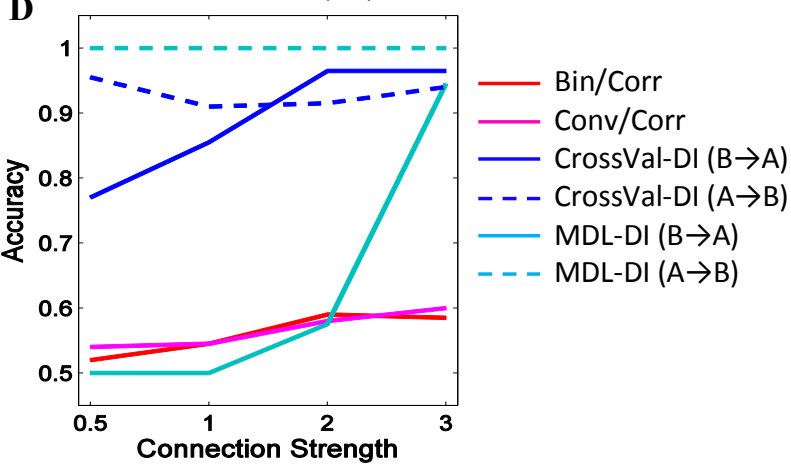

Fig. 2. (A) Sample spike trains for two-neuron topology. (B) Distribution of $\mathrm{p}$-values obtained over 100 simulations for one set of parameters. Red line indicates threshold of significance $p=0.05$. (C) Distribution of directed information values determined using CrossVal-DI and MDL-DI for both directions. (D) Accuracy of each metric at different connection strengths for one set of parameters

we varied the strength of the connection from 0.5 to 3 times the original value. For each configuration, we generated 100 sets of $200,000 \mathrm{~ms}$ spike trains in which we computed the firing rate correlation and directed information. We also generated independent ("null" case) spike trains to test for false positives. The firing rate used in the correlation analysis is estimated in two ways: $100 \mathrm{~ms}$ binning ("Bin/Corr"), or convolved with a $100 \mathrm{~ms}$ window ("Conv/Corr"). To determine if a correlation is significant, we follow standard procedure and transform the correlation to a t-statistic, in which significance is set at $p<0.05$. The directed information is estimated using minimum description length (refer to as "MDL-DI") or with smoothness constraint and cross-validation (CrossVal-DI).

\section{RESUlts}

\section{A. Two-neuron simulations}

For each simulation configuration, we measured the overall accuracy (fraction of true positives plus true negatives) of each metric. For directed information, we also computed the measure for the opposite direction, wherein the accuracy was simply the fraction of true negatives observed. The results for one of the parameter sets at varying connection strengths are shown in Fig. 2. As expected, the accuracy of all four metrics went up as the connection strength increased. Since the accuracy in general can fluctuate for different parameter sets, we normalize the accuracy values by computing the percent increase over the lowest accuracy of the four metrics at the lowest connection strength for each parameter set. The percent increase for each metric averaged across all 6 parameter sets are shown in Table 1. For the DI methods, only B to A is included. At all connection strengths, CrossVal-DI consistently had the highest overall accuracy. Although Conv/Corr had very high true positive rates (Fig. 2B), it also reported a high false positive rate. Conversely, MDL-DI consistently underestimated the significance of the connections, resulting in high true negative rates, but poor true positive detections.

TABLE 1

PERCENT INCREASE IN ACCURACY AVERAGED ACROSS PARAMETER SETS

\begin{tabular}{|ccccc|}
\hline Strength & Bin/Corr & Conv/Corr & CrossVal-DI & MDL-DI \\
\hline 0.5 & $0.03 \pm 0.03$ & $0.07 \pm 0.05$ & $\mathbf{0 . 2 5} \pm \mathbf{0 . 2}$ & 0 \\
1 & $0.22 \pm 0.2$ & $0.13 \pm 0.07$ & $\mathbf{0 . 5 8} \pm \mathbf{0 . 2}$ & $0.03 \pm 0.07$ \\
2 & $0.51 \pm 0.4$ & $0.16 \pm 0.08$ & $\mathbf{0 . 8 3} \pm \mathbf{0 . 1}$ & $0.31 \pm 0.4$ \\
3 & $0.61 \pm 0.4$ & $0.21 \pm 0.01$ & $\mathbf{0 . 9 1} \pm \mathbf{0 . 0 5}$ & $0.80 \pm 0.4$ \\
\hline
\end{tabular}

\section{B. Three-neuron simulations}

\section{1) Proxy topology}

We tested the ability of the metrics to detect indirect connections where a neuron $\mathrm{B}$ influences the activity of neuron $\mathrm{C}$ via another neuron $\mathrm{A}$. The accuracy and raw number of significant detections found at varying connection strengths are shown in Fig. 3A-B. For all four metrics, the number of indirect connections detected increased with connection strength. As expected, the directed information in the opposite direction (from $\mathrm{C}$ to $\mathrm{B}$ ) measured using CrossVal-DI aptly remained around the null case level of significance at all connection strengths (although MDL-DI also detected no significant connections in this direction, this is likely due to the method's high threshold for a positive detection).

\section{2) Cascading topology}

We also considered another topology where both neurons $\mathrm{A}$ and $\mathrm{C}$ are influenced by neuron B. Since it is unclear in this topology whether the indirect connections between $\mathrm{A}$ and C are "true" or not, we simply analyzed the number of 
A
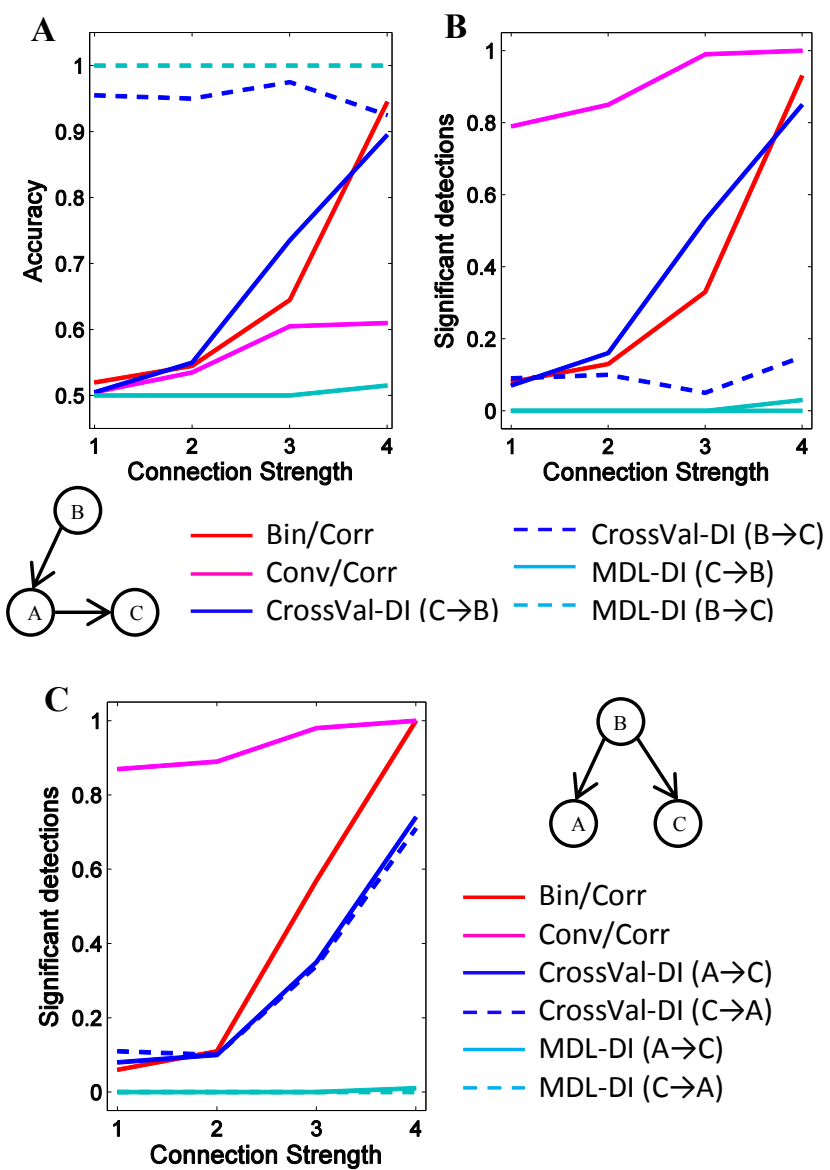

Fig. 3. (A) Accuracy and (B) percentage of significant detections for proxy topology. (C) Percentage of significant detections for cascading topology.

significant connections as the connection strength increased. In this topology, the directed information (measured using CrossVal-DI) from $\mathrm{A}$ to $\mathrm{C}$ and $\mathrm{C}$ to $\mathrm{A}$ both increased with connection strength. Directed information estimated using MDL-DI did not reveal any significant connections. Using correlations, we see the exact same trend as seen for the indirect connections for the proxy topology.

\section{Discussion}

In this paper, we assessed the performance of directed information for inferring connectivity compared to the conventional method of using correlations. We demonstrate that estimating directed information using the CrossVal-DI technique consistently provides the highest level of accuracy across different parameter sets and connection strengths. Furthermore, CrossVal-DI is able to correctly identify the direction of influence, which cannot be determined with correlations. Most importantly, although CrossVal-DI imposes an a priori model for the causal influence, the method is still able to robustly detect connections even when the underlying model is not a perfect match, but contains different non-linearities and terms that are not accounted for in the a priori model. This robustness is also reflected in the proxy topology where the connection between the two neurons is further obscured by an intermediate neuron.
Again, CrossVal-DI can still accurately detect the direction of influence. One limitation of directed information is in the case of a cascading topology. In this topology, significant connections are found using CrossVal-DI between two neurons $\mathrm{A}$ and $\mathrm{C}$ that are influenced by a common neuron $\mathrm{B}$, even though there is technically no causal influence between the two. Unfortunately, this is in an inherent drawback of causality models based on Granger's causality. In theory, the dependence between $\mathrm{A}$ and $\mathrm{C}$ can be removed by conditioning the distributions on the activity of $\mathrm{B}$. In practice, however, this may not be feasible since there can be many possible choices of neurons to condition on. Future work will be needed to apply directed information to experimental data to explore whether the additional directional information gained using directed information can provide new insights beyond correlation analysis.

\section{REFERENCES}

[1] L. Lee, L.M. Harrison, and A. Mechelli, "A report of the functional connectivity workshop, Dusseldorf 2002," NeuroImage, vol. 19, Jun. 2003, pp. 457-465.

[2] V.M. Eguíluz, D.R. Chialvo, G.A. Cecchi, M. Baliki, and A.V. Apkarian, "Scale-free brain functional networks," Physical Review Letters, vol. 94, Jan. 2005, p. 018102.

[3] G. Pfurtscheller and C. Andrew, "Event-Related changes of band power and coherence: methodology and interpretation," Journal of Clinical Neurophysiology: Official Publication of the American Electroencephalographic Society, vol. 16, Nov. 1999, pp. 512-519.

[4] L. Paninski, "Estimation of entropy and mutual information," Neural Computation, vol. 15, Jun. 2003, pp. 1191-1253.

[5] A. Mcintosh and F. Gonzalez-Lima, "Structural equation modeling and its application to network analysis in functional brain imaging," Human Brain Mapping, vol. 2, 1994, pp. 2-22.

[6] K.J. Friston, L. Harrison, and W. Penny, "Dynamic causal modelling," NeuroImage, vol. 19, Aug. 2003, pp. 1273-1302.

[7] B.P. Rogers, V.L. Morgan, A.T. Newton, and J.C. Gore, "Assessing Functional Connectivity in the Human Brain by FMRI," Magnetic resonance imaging, vol. 25, Dec. 2007, pp. 1347-1357.

[8] M. Kamiński, M. Ding, W. Truccolo, and S. Bressler, "Evaluating causal relations in neural systems: granger causality, directed transfer function and statistical assessment of significance.," Biological cybernetics, vol. 85, 2001, pp. 145-157.

[9] C. Quinn, T. Coleman, N. Kiyavash, and N. Hatsopoulos, "Estimating the directed information to infer causal relationships in ensemble neural spike train recordings," Journal of Computational Neuroscience, vol. 30, Jun. 2010, pp. 17-44.

[10] P. Amblard and O.J.J. Michel, "On directed information theory and Granger causality graphs," Journal of Computational Neuroscience, vol. 30, 2010, pp. 7-16.

[11] J.L. Massey, "Causality, Feedback And Directed Information," Proceedings of the International Symposium on Information Theory and its Applications, 1990, pp. 303-305.

[12] W. Truccolo, U.T. Eden, M.R. Fellows, J.P. Donoghue, and E.N. Brown, "A point process framework for relating neural spiking activity to spiking history, neural ensemble, and extrinsic covariate effects," Journal of Neurophysiology, vol. 93, 2005, p. 1074.

[13] J.W. Pillow, J. Shlens, L. Paninski, A. Sher, A.M. Litke, E.J. Chichilnisky, and E.P. Simoncelli, "Spatio-temporal correlations and visual signalling in a complete neuronal population," Nature, vol. 454,2008 , pp. 995-999.

[14] W. Truccolo, L.R. Hochberg, and J.P. Donoghue, "Collective dynamics in human and monkey sensorimotor cortex: predicting single neuron spikes," Nature Neuroscience, 2009.

[15] D. Song, R.H. Chan, V.Z. Marmarelis, R.E. Hampson, S.A. Deadwyler, and T.W. Berger, "Sparse generalized LaguerreVolterra model of neural population dynamics," Engineering in Medicine and Biology Society, 2009. EMBC 2009. Annual International Conference of the IEEE, 2009, pp. 4555-4558. 